$\mathrm{R} / 94 / 44$

9501018

December, 1994

\title{
Supersymmetric spacetimes in $(2+1)$ adS-supergravity models
}

\author{
J.M. IZQUIERDO AND P.K. TOWNSEND \\ DAMTP, Univ. of Cambridge, Silver St., Cambridge, U.K.
}

\begin{abstract}
We find a class of $(2+1)$-dimensional spacetimes admitting Killing spinors appropriate to $(2,0)$ adS-supergravity. The vacuum spacetimes include anti-de Sitter (adS) space and charged extreme black holes, but there are many others, including spacetimes of arbitrarily large negative energy that have only conical singularities, and the spacetimes of fractionally charged point particles. The non-vacuum spacetimes are those of self-gravitating solitons obtained by coupling $(2,0)$ adS supergravity to sigma-model matter. We show, subject to a condition on the matter currents (satisfied by the sigma model), and a conjecture concerning global obstructions to the existence of certain types of spinor fields, that the mass of each supersymmetric spacetime saturates a classical bound, in terms of the angular momentum and charge, on the total energy of arbitrary field configurations with the same boundary conditions, although these bounds may be violated quantum mechanically.
\end{abstract}




\section{Introduction}

Theories of gravity in $2+1$ dimensions continue to be a fertile area for the investigation of the consequences of general covariance in field theory. The $(\mathrm{p}, \mathrm{q})$ anti-de Sitter (adS) supergravity theories [1], which can be viewed as pure ChernSimons (CS) terms for the superalgebras $\operatorname{osp}(p \mid 2 ; \mathbb{R}) \oplus \operatorname{osp}(q \mid 2 ; \mathbb{R})$, are of particular interest for various reasons. Firstly, the $(0,0)$ case is just $2+1$ Einstein gravity with a negative cosmological constant, which has been shown to admit asymptoticallyadS black hole solutions [2]. This theory is the bosonic sector of the $(1,1)$ theory and in this context one can ask whether black hole solutions are 'supersymmetric' in the sense of preserving some of the supersymmetry of the adS vacuum. This is equivalent to asking whether the black hole spacetime admits a suitably-defined Killing spinor and it has been shown [3] that only the extreme rotating black holes, and the non-rotating 'black hole vacuum' [2], do so. Secondly, for $p$ or $q$ greater than one, the action includes an $s o(p) \oplus s o(q)$ pure CS term. The simplest such case is the $(2,0)$ theory which we review below. We find a one-function class of 'off-shell' bosonic field configurations of this model that preserve some supersymmetry, i.e. admit Killing spinors. The function is fixed by the field equations and the boundary conditions, and depends on the adS inverse radius, $m$. For zero charge, $Q$, we find a class of supersymmetric vacuum spacetimes with ADM mass $M=2 m J-n^{2}$ (relative to the 'black hole vacuum'), and total angular momentum $J$, for any integer $n$. When $n=0$ or $|n|=1$ we recover, for $J=0$, the black-hole vacuum and anti-de Sitter spacetime respectively. For $|n|>1$ we find a new class of supersymmetric spacetimes with a naked conical singularity of negative deficit angle $\delta=-2 \pi(|n|-1)$. The point particle spacetimes [4] with $-1>M>0$, which

have a naked conical singularity of positive deficit angle $\delta=2 \pi(1+\sqrt{M})$, are also supersymmetric when $Q$ is such that $M=-4 Q^{2}$.

It is a general feature of supergravity theories that solutions that are supersymmetric (in the above sense of admitting a Killing spinor) saturate a BogomolnyiGibbons-Hull type of bound on the ADM energy of any field configuration that is 
either non-singular or, if singular, can evolve from an initially non-singular configuration. A special case of this bound is the positivity of the ADM energy. One usually finds (e.g. for asymptotically flat spacetimes of dimension $d>3$ ) that, once boundary conditions at spatial infinity have been specified, there is a unique supersymmetric zero charge state which is naturally identified as the vacuum. An exception to this rule is $(2,0)(a d S)_{3}$-supergravity because it turns out that there are many supersymmetric zero charge configurations. The only non-singular one is the adS spacetime itself but the black hole vacuum, which has a null singularity, is also supersymmetric and is non-singular on all spacelike hypersurfaces orthogonal to the orbits of the timelike Killing vector field. Other supersymmetric zero charge spacetimes have naked timelike conical singularities and do not admit a non-singular Cauchy surface, so only adS spacetime and the black hole vacuum are candidates for 'the' vacuum. The reason that there can be two such candidates is that in $2+1$ dimensions spatial infinity is not simply connected so that, as appreciated in [3], spinors that are asymptotically Killing may be either periodic or anti-periodic at spatial infinity. However, knowledge of the behaviour at spatial infinity of an asymptotically Killing spinor requires more than the knowledge of whether it is periodic or antiperiodic; one must know its phase, which is specified by the integer $n$. We shall shortly return to the significance of this fact.

A variant [5] of the ADM method can be used to find expressions as surface integrals of mass, angular momentum and charge in asymptotically adS spacetimes of arbitrary dimension. In three dimensions a simplification of this method is made possible by the fact that three-dimensional adS (super)gravity can be viewed as a pure Chern-Simons theory $[1,6]$. This leads naturally to a definition of mass and other 'charges' in terms of holonomy, in agreement with other methods $[2,7]$. We use this result and the methods of $[8,9]$ to deduce that the energy $E=M+1$ of an asymptotically adS spacetime is bounded in terms of its angular momentum and charge. Specifically, for zero angular momentum and charge one finds that $M \geqslant-n^{2}$, where the integer $n$ specifies the type of asymptotically Killing spinor under consideration. The proof of this bound depends on the asymptotically 
Killing spinor being non-singular on a Cauchy surface and satisfying a Dirac-like equation (the 'Witten condition'). It can happen that a particular asymptotically adS spacetime will not admit such a spinor for certain values of $n$. An example is adS spacetime itself for which $|n|=1$ is possible but $n=0$ is not; if it were we could prove that $M \geqslant 0$ for this spacetime whereas in fact $M=-1$. We shall later provide some evidence for the conjecture that, for any asymptotically adS solution of the Einstein equations (with suitably well-behaved matter) for which there exists a non-singular Cauchy surface, it is possible to find an asymptotically Killing spinor satisfying the Witten condition with either $n=0$ or $|n|=1$. If this is true then we need consider only $n=0$ and $|n|=1$, since $|n|>1$ leads to a weaker bound. This would establish the absolute stability of adS spacetime, but the black hole vacuum might be unstable against semi-classical tunnelling. An instability of this type has been shown to afflict the Kaluza-Klein vacuum of fivedimensional general relativity [10], for which the positive energy theorem fails for similar reasons.

This conjecture does not imply the non-existence of spacetimes with $M<-1$; as already mentioned, there is a supersymmetric spacetime with $M=-n^{2}$ for every integer $n$. However, the supersymmetric spacetimes with $|n|>1$ have naked singularities that prevent one from finding a non-singular Cauchy surface and this may be a feature of all solutions of the field equations with $M<-1$, for sufficiently well-behaved matter. We remark that spacetimes with naked singularities should not be considered unphysical per se. It is known that cosmic censorship is false for asymptotically $(a d S)_{3}$ spacetimes since non-singular matter can collapse to a naked singularity if $0>M>-1[11]$. The singularity in this case is a conical one with a positive deficit angle. We suggest that the appropriate extension of the cosmic censorship hypothesis in this context is such as to permit naked singularities of this type but no others.

In certain respects, $(2+1)$-dimensional supergravity is similar to $(4+1)$ dimensional supergravity for which it is known [9] that supersymmetric black holes can be viewed as limits of self-gravitating solitons of supergravity coupled to matter. 
One of the motivations for the work reported in this paper was to investigate whether a similar result holds for self-gravitating solitons of the $2+1$ dimensional $(2,0)$ adS supergravity coupled to matter. In addressing this question our first task is the construction of a suitable coupling of matter to $(2,0)$ adS supergravity. The problem of coupling scalar multiplets to $N$-extended Einstein supergravity without a cosmological constant was solved in [12]. The inclusion of a cosmological term is straightforward for $N=1$ (leading to a matter coupled $(1,0)$ adS supergravity theory), and is implicit in the $N=1$ superspace results of [13]. In contrast, the inclusion of a cosmological term for $N>1$, in the presence of matter, cannot be deduced simply from the results of [12] because the field content differs by the presence of the Chern-Simons gauge field(s). Taking the $N=2$ flat space sigmamodel as our starting point we construct a locally supersymmetric coupling of this action to $(2,0)$ adS supergravity. The target space of this locally-supersymmetric sigma model is Kähler. We choose it to be compact, in order to allow for the possibility of sigma-model solitons, and we study the particular case of the Riemann sphere in detail. In the absence of supergravity the soliton solutions are simply holomorphic functions on 2-space considered as the complex plane (see, for example, [14]). Remarkably, this continues to be true, for an appropriate choice of complex coordinate, even when the gravitational corrections are included, although the holomorphic functions must now satisfy a further restriction. For the case of an $S^{2}$ target space this further restriction is that the holomorphic function be homogeneous. These self-gravitating solitons admit Killing spinors and saturate an energy bound. In this respect, self-gravitating solitons of three-dimensional supergravity/matter theories are similar to those of five-dimensional supergravity [9], but the Einstein equation is now a non-linear second-order ODE. Although we have not been able to solve this equation analytically, we argue that the solitons governed by this equation are non-singular. We are also able to determine their asymptotic behaviour; we find that the solitons always have a logarithmic dependence on the natural radial coordinate, for large radius, but such that $E-2 m J$ is well-defined, and bounded in terms of the charge $Q$, even though neither $E$ nor $J$ 
is separately well-defined. Thus, the asymptotic symmetry group of these solutions is a proper subgroup of the adS group.

We conclude this introduction with a brief account of the pure $(2,0)$ adS supergravity [1]. The field content consists of the metric tensor, $g_{\mu \nu}$, a complex Rarita-Schwinger field, $\psi_{\mu}$, and an Abelian gauge field $A_{\mu}$. The action is

$$
S=\int d^{3} x\left[-\frac{1}{2} e R+\frac{i}{2} \varepsilon^{\mu \nu \rho} \bar{\psi}_{\mu} \mathcal{D}_{\nu} \psi_{\rho}-2 m \varepsilon^{\mu \nu \rho} A_{\mu} \partial_{\nu} A_{\rho}+4 m^{2} e\right]
$$

where $m$ is a constant with units of mass which we may assume to be positive. The covariant derivative $\mathcal{D}$ is defined by

$$
\mathcal{D}_{\mu}=D_{\mu}+2 i m A_{\mu}-i m \gamma_{\mu}
$$

where $D$ is the usual Lorentz-covariant derivative except that the spin connection has the non-zero torsion

$$
T_{\mu \nu}^{a} \equiv D_{[\mu} e_{\nu]}^{a}=\frac{1}{4} \bar{\psi}_{\mu} \gamma^{a} \psi_{\nu}
$$

The action (1.1) is invariant under the supersymmetry transformations

$$
\begin{aligned}
\delta_{\epsilon} e_{\mu}{ }^{a} & =\frac{1}{4} \bar{\epsilon} \gamma^{a} \psi_{\mu}+\text { c.c. } \\
\delta_{\epsilon} \psi_{\mu} & =\mathcal{D}_{\mu} \epsilon \\
\delta_{\epsilon} A_{\mu} & =\frac{1}{4}\left(\bar{\epsilon} \psi_{\mu}+\text { c.c. }\right) .
\end{aligned}
$$

We follow the conventions of [1], i.e. the metric signature is 'mostly minus' and the gamma matrices $\gamma^{a}$ are pure imaginary. 


\section{Killing Spinors}

The condition for a purely bosonic field configuration of $(2,0)$ adS supergravity to preserve at least one supersymmetry of $(2,0)$ adS supergravity is that the equation

$$
\delta_{\epsilon} \psi_{\mu}=0
$$

admit a non-zero solution for the spinor parameter $\epsilon$. Since this equation is linear in $\epsilon$ its consequences are unchanged if $\epsilon$ is replaced by the commuting spinor $\kappa$. Making this replacement, we have to solve

$$
\mathcal{D}_{\mu} \kappa=0
$$

for non-zero $\kappa$.

An alternative form for the covariant derivative $\mathcal{D}$ is

$$
\mathcal{D}_{\mu}=\partial_{\mu}+i B_{\mu}{ }^{a} \gamma_{a}+2 i m A_{\mu},
$$

where

$$
B_{\mu}{ }^{a}=\frac{1}{4} \varepsilon^{a b c} \omega_{\mu b c}-m e_{\mu}{ }^{a}
$$

is an $S p(2 ; \mathbb{R}) \cong S l(2 ; \mathbb{R})$ gauge field. This is a reflection of the fact that $(a d S)_{3}$ gravity can be viewed as a Chern-Simons theory for the adS group $S O(2,2) \cong$ $S l(2 ; \mathbb{R}) \times S l(2 ; \mathbb{R})[1]$, and that for the extension to $(\mathrm{p}, 0)$ supergravity only one $S l(2 ; \mathbb{R})$ factor is supersymmetrized. Since the torsion vanishes in a purely bosonic background, the $S l(2 ; \mathbb{R})$ gauge field $B$ can be expressed entirely in terms of the dreibein as follows:

$$
B_{\mu}{ }^{a}=\frac{1}{4} \varepsilon^{a b c}\left[2 e_{b}^{\nu} \partial_{[\mu} e_{\nu] c}-e_{b}^{\nu} e_{c}^{\rho} \partial_{\nu} e_{\rho}^{d} e_{\mu d}\right]-m e_{\mu}^{a} .
$$


Thus, Killing spinors $\kappa$ are non-zero solutions of

$$
\left(\partial_{\mu}+i B_{\mu}^{a} \gamma_{a}+2 i m A_{\mu}\right) \kappa=0
$$

with $B_{\mu}{ }^{a}$ as given above. This equation has the integrability condition

$$
\left(m G^{\mu}+S^{\mu}{ }_{a} \gamma^{a}\right) \kappa=0
$$

where

$$
\begin{aligned}
S^{\mu}{ }_{a} & \equiv \varepsilon^{\mu \nu \rho}\left(\partial_{\nu} B_{\rho a}-\varepsilon_{a b c} B_{\nu}{ }^{b} B_{\rho}^{c}\right) \\
G^{\mu} & \equiv 2 \varepsilon^{\mu \nu \rho} \partial_{\nu} A_{\rho}
\end{aligned}
$$

are the duals of the $S l(2 ; \mathbb{R})$ and $U(1)$ field strengths respectively.

In the absence of matter the Euler-Lagrange equations for the bosonic fields of $(2,0)$ adS supergravity are simply

$$
S^{\mu}{ }_{a}=0 \quad G^{\mu}=0
$$

so that (2.7) is automatically satisfied by solutions of the source-free equations. Since only the $(a d S)_{3}$ spacetime with zero $U(1)$ charge admits the full complement of Killing spinors, i.e. four for $(2,0)$ supergravity (this being the real dimension of the space of complex two-component spinors) we shall seek metric and gauge field configurations admitting two Killing spinors ${ }^{\star}$. We can implement this by requiring $\kappa$ to satisfy a condition of the form

$$
\left(1-\gamma^{a} b_{a}\right) \kappa=0
$$

for some complex functions $b_{a}$. Provided that

$$
b \cdot b=1
$$

this condition projects out half the components of $\kappa$. This constraint on $\kappa$ can be

\footnotetext{
$\star$ This will of course include the zero charge anti-de Sitter spacetime as a special case.
} 
solved by writing

$$
\kappa=N\left(1+\gamma^{a} b_{a}\right) \zeta_{0}
$$

for arbitrary complex function $N$ and constant spinor $\zeta_{0}$. When (2.12) is substituted into the integrabilty condition (2.7) one finds that

$$
S^{\mu}{ }_{a}+m G^{\mu} b_{a}+i \varepsilon_{a b c} b^{b} S^{\mu b}=0 .
$$

This is of course satisfied by any solution of the empty space equations, but we wish to find off-shell configurations that admit Killing spinors. When we subsequently consider the implications of the field equations our Killing spinor results will then be equally applicable whether we consider the field equations with or without matter. Note that the integrability condition (2.7) is necessary but insufficient for the background to admit a Killing spinor, so after analysing the content of the integrability conditions we must then return to the Killing spinor equation (2.6), which will restrict the function $N$ in (2.12).

Rather than attempt to find the general solution of (2.6) we shall find solutions by making the following ansatz for the frame one-forms $e^{a}$ :

$$
e^{\underline{0}}=f(r) d t \quad e^{\underline{1}}=h(r) d r \quad e^{\underline{\underline{2}}}=\frac{u(r)}{r} d t+r d \varphi,
$$

where underlining indicates a frame index (i.e. $a=\underline{0}, \underline{1}, \underline{2}$ ). The metric is then given by

$$
d s^{2}=\left[f^{2}-\left(\frac{u}{r}\right)^{2}\right] d t^{2}-2 u d t d \varphi-h^{2} d r^{2}-r^{2} d \varphi^{2} .
$$

This ansatz is suggested by the fact that the function $u(r)$ is constant for the black hole. The $\operatorname{Sl}(2, \mathbb{R})$ one-form potential $B_{a}$ may now be computed from (2.5) and 
the result is

$$
\begin{aligned}
B_{\underline{0}}= & \left(\frac{u^{\prime}}{4 r h}-m f\right) d t+\frac{1}{2 h} d \varphi \\
B_{\underline{1}}= & {\left[\frac{1}{4 r f}\left(\frac{2 u}{r}-u^{\prime}\right)+m h\right] d r } \\
B_{\underline{2}}= & {\left[-\frac{1}{4 h}\left(2 f^{\prime}-\frac{u u^{\prime}}{r^{2} f}+\frac{2 u^{2}}{r^{3} f}\right)+\frac{m u}{r}\right] d t } \\
& +\left[\frac{1}{4 f h}\left(u^{\prime}-\frac{2 u}{r}\right)+m r\right] d \varphi .
\end{aligned}
$$

Our ansatz may now be shown to imply that certain components of $S^{\mu}{ }_{a}$ vanish. Specifically, one finds that

$$
S_{\underline{0}}^{r}=S_{\underline{2}}^{r}=S_{\underline{1}}^{t}=S_{\underline{1}}^{\varphi}=0
$$

We now separate the integrability condition (2.13) into its various components. Firstly we have

$$
S^{r}=0
$$

which, since $B_{\mu}{ }^{a}$ depends only on $r$, is equivalent to

$$
B_{\varphi \underline{2}} B_{t \underline{0}}=B_{\varphi \underline{0}} B_{t \underline{2}}
$$

Secondly, we have

$$
m G^{\mu}+S^{\mu}{ }_{a} b^{a}=0
$$

and finally

$$
\begin{gathered}
\left(1-b_{\underline{0}}^{2}\right) S^{t \underline{0}}+\left(i b_{\underline{1}}-b_{\underline{2}} b_{\underline{0}}\right) S^{t \underline{2}}=0 \\
\left(1-b_{\underline{0}}^{2}\right) S^{\varphi \underline{0}}+\left(i b_{\underline{1}}-b_{\underline{2}} b_{\underline{0}}\right) S^{\varphi \underline{2}}=0
\end{gathered}
$$

We now return to the Killing spinor equation. Given that $\kappa$ has the form of 
(2.12), we find by substitution into (2.6) that

$$
2 m A_{\mu}-i \partial_{\mu}(\ln N)+b_{a} B_{\mu}^{a}=0
$$

and

$$
B_{\mu}^{a}-i \varepsilon^{a b c} b_{b} B_{\mu c}-i \partial_{\mu} b^{a}-b^{a} b_{c} B_{\mu}^{c}=0
$$

Observe now that (2.19) implies

$$
\begin{aligned}
& B_{t \underline{2}}=\Lambda(r) B_{\varphi \underline{2}} \\
& B_{t \underline{0}}=\Lambda(r) B_{\varphi \underline{0}},
\end{aligned}
$$

for some function $\Lambda$ of $r$, which must be finite since $B_{\varphi \underline{0}} \neq 0$. It follows that the $t$ and $\varphi$ components of (2.23) are consistent only if

$$
\left(\partial_{t}-\Lambda \partial_{\varphi}\right) b_{a}=0
$$

Differentiating this equation with respect to $r$, and denoting this derivative by a prime, we find, on the one hand, that

$$
\left(\partial_{t}-\Lambda \partial_{\varphi}\right) b_{a}^{\prime}=\Lambda^{\prime} \partial_{\varphi} b_{a}
$$

On the other hand, the $r$ component of (2.23) yields

$$
\begin{aligned}
& i b_{\underline{0}}^{\prime}=B_{r \underline{1}}\left(i b_{2}+b_{\underline{0}} b_{1}\right) \\
& i b_{\underline{1}}^{\prime}=B_{r \underline{1}}\left(1+b_{\underline{1}}^{2}\right) \\
& i b_{\underline{2}}^{\prime}=B_{r \underline{1}}\left(i b_{\underline{0}}+b_{\underline{1}} b_{2}\right),
\end{aligned}
$$

which we may use to eliminate $b_{a}^{\prime}$ in $(2.27)$ in terms of $b_{a}$ and $B_{r \underline{1}}$. Since $B_{r \underline{1}}$ depends only on $r$, and $b_{a}$ satisfies (2.25), the left hand side of (2.26) vanishes, and we conclude that

$$
\Lambda^{\prime} \partial_{\varphi} b_{a}=0
$$

This leads to two fundamentally different ways of solving the Killing spinor equation: 


\section{Either}

(i) $\Lambda$ is constant, in which case $b$ depends on $t$ and $\phi$ only through the combination $\hat{\varphi} \equiv \varphi+\Lambda t$, i.e.

$$
b_{a}=b_{a}(\hat{\varphi}, r)
$$

or

(ii) $b_{a}$ is independent of both $t$ and $\varphi$, i.e.

$$
b_{a}=b_{a}(r)
$$

and $\Lambda(r)$ is unrestricted.

Option (i) is the more difficult to analyse, partly because there is in this case a further bifurcation of possibilities according to whether or not $\partial_{\varphi} b_{2}$ vanishes. It turns out that all supersymmetric spacetimes found via option (i) are also found via option (ii); that this is possible is due to the fact that the function $N$ can be $\varphi$-dependent. The converse is not true, however; option (ii) leads to some supersymmetric spacetimes that cannot be found via option (i). We shall therefore omit the analysis of option (i) and proceed with option (ii).

For either option, the $t$ and $\varphi$ components of (2.23) are equivalent so we need consider only the latter. Since $\partial_{\varphi} b_{a}=0$ for option (ii) these reduce to the algebraic equations

$$
\begin{aligned}
B_{\varphi \underline{\underline{0}}}\left(1-b_{\underline{0}}^{2}\right)+B_{\varphi \underline{\underline{2}}}\left(b_{\underline{\underline{0}}} b_{\underline{2}}-i b_{\underline{1}}\right) & =0 \\
B_{\varphi \underline{0}}\left(i b_{\underline{2}}-b_{\underline{1}} b_{\underline{0}}\right)+B_{\varphi \underline{\underline{2}}}\left(b_{\underline{1}} b_{\underline{2}}-i b_{\underline{0}}\right) & =0 \\
B_{\varphi \underline{\underline{0}}}\left(i b_{\underline{1}}+b_{\underline{0}} b_{\underline{2}}\right)-B_{\varphi \underline{\underline{2}}}\left(1+b_{\underline{2}}^{2}\right) & =0 .
\end{aligned}
$$

Solving the last of these equations for $b_{1}$ and substituting it into the other two 
equations we find that

$$
\begin{aligned}
& b_{\underline{0}}=R b_{2} \pm \sqrt{1-R^{2}} \\
& b_{\underline{1}}=-i\left[R \mp b_{\underline{2}} \sqrt{1-R^{2}}\right],
\end{aligned}
$$

where

$$
R=\frac{B_{\varphi \underline{2}}}{B_{\varphi \underline{0}}}=\frac{1}{2 f}\left(u^{\prime}-\frac{2 u}{r}\right)+2 m h r .
$$

Substituting this result into the equations (2.27) one discovers that $R$ and $b_{\underline{2}}$ satisfy the ordinary differential equations

$$
R^{\prime}-2\left(1-R^{2}\right) B_{r \underline{1}}=0
$$

and

$$
b_{\underline{2}}^{\prime}= \pm B_{r \underline{1}} \sqrt{1-R^{2}}\left(1+b_{\underline{2}}^{2}\right) .
$$

The equation for $R$ together with (2.19) is equivalent to the following two equations that determine the functions $f$ and $h$ in terms of $u$ and two constants $\alpha$ and $\beta$ :

$$
\begin{aligned}
4 m h f & =2 \alpha-\frac{u^{\prime}}{r} \\
f^{2} & =\left(\alpha r-\frac{u}{r}\right)^{2}+\beta^{2} .
\end{aligned}
$$

The constant $\alpha$ must be real and cannot vanish if the spacetime is to be asymptotic to anti-de Sitter space (or the black hole vacuum). By a redefinition of the time coordinate $t$, we can effectively change $\alpha$ to any non-zero value, and the choice

$$
\alpha=2 m
$$

is convenient. The constant $\beta^{2}$ must also be real. This would, in principle, allow $\beta$ to be pure imaginary but we shall seee shortly that $\beta$ must be real too. 
One can now deduce the following useful formulae:

$$
\begin{aligned}
R=\frac{\sqrt{f^{2}-\beta^{2}}}{f} & \sqrt{1-R^{2}}=\frac{\beta}{f} \\
B_{r \underline{1}}=\frac{1}{2 f}\left(2 m r-\frac{u}{r}\right)^{\prime} & \Lambda \equiv \frac{B_{t \underline{0}}}{B_{\varphi \underline{0}}}=\frac{u^{\prime}}{r}-2 m .
\end{aligned}
$$

Using these formulae one can easily solve (2.35) for $b_{2}$ and simplify the expressions for the other components of $b_{a}$. The result is

$$
\begin{aligned}
& b_{\underline{0}}=\frac{\sqrt{f^{2}-\beta^{2}}}{f} b_{2}+\frac{\beta}{f} \\
& b_{\underline{1}}=-i\left(\frac{\sqrt{f^{2}-\beta^{2}}}{f}-\frac{\beta}{f} b_{2}\right) \\
& b_{\underline{2}}=\frac{k_{+} \sqrt{f+\beta}+k_{-} \sqrt{f-\beta}}{k_{-} \sqrt{f+\beta}-k_{+} \sqrt{f-\beta}}
\end{aligned}
$$

where $k_{ \pm}$are arbitrary constants.

We now turn to the equation for the one-form $A$, (2.22). If the associated abelian gauge group is $\mathbb{R}$ then the gauge freedom of $A$ can be used to remove the phase of $N$. However, if the gauge group is $U(1)$ then we must distinguish between 'small' and 'large' gauge transformations, i.e. between elements of $U(1)$ that are connected to the identity and those which are not connected to the identity. Because the action for the $U(1)$ gauge field $A$ is $m$ times the CS term, the charge $Q$ associated with $A$ is

$$
Q=\frac{m}{\pi} \oint_{\infty} A
$$

as will be explained in more detail in section 4. A large gauge transformation will change the value of this charge, so fixing the charge restricts the gauge freedom to small gauge transformations. In this case the phase of $N$ cannot necessarily be completely removed by a gauge transformation but we can use the freedom of 
small gauge transformations to bring the function $N$ to the form

$$
N=a(r, \varphi) e^{\frac{i n}{2} \varphi+i m \beta t}
$$

where $n$ is an integer and $a(r, \varphi)$ is a real function. The t-dependent term in the phase is chosen for later convenience. Taking real and imaginary parts of (2.22) now determines the one-form $A$ and the function $a(r, \varphi)$. It is at this point that one discovers that $\beta$ must be purely real rather than purely imaginary because $a(r, \varphi)$ would otherwise be a non-periodic function of $\varphi$. For real $\beta$ one finds that

$$
A=-\left(\frac{\beta u^{\prime}}{2\left(4 m r-u^{\prime}\right)}\right) d t-\left(\frac{\beta r}{4 m r-u^{\prime}}+\frac{n}{4 m}\right) d \varphi
$$

and

$$
a=a(r) \equiv k_{-} \sqrt{f+\beta}-k_{+} \sqrt{f-\beta}
$$

Combining (2.12) with (2.39) now yields the following result for the Killing spinor $\kappa$ :

$$
\begin{aligned}
& \kappa=e^{\frac{i n}{2} \varphi+i m \beta t}\left[k_{-} \sqrt{f+\beta}-k_{+} \sqrt{f-\beta}\right] \\
& \times\left\{\left[1+\frac{1}{f}\left(\beta \gamma^{\underline{0}}-i \sqrt{f^{2}-\beta^{2}} \gamma^{\underline{1}}\right)\right]+b_{\underline{2}} \gamma^{\underline{2}}\left[1-\frac{1}{f}\left(\beta \gamma^{\underline{0}}-i \sqrt{f^{2}-\beta^{2}} \gamma^{\underline{1}}\right)\right]\right\} \zeta_{0} .
\end{aligned}
$$

It is convenient to normalize the constant spinor $\zeta_{0}$ such that

$$
\zeta_{0}^{\dagger} \zeta_{0}=1
$$

In addition, we may require without loss of generality that $\zeta_{0}$ satisfy $P \zeta_{0}=\zeta_{0}$ for some constant projection matrix, $P$, that projects out two components of $\zeta_{0}$, since (for fixed $k_{ \pm}$) the real dimension of the space of Killing spinors $\kappa$ is two, not 
four, despite the fact that $\zeta_{0}$ has two complex, and hence four real, components. A convenient choice is

$$
\left[k_{+}^{2}+k_{-}^{2}\right]^{-1}\left[\left(k_{-}^{2}-k_{+}^{2}\right) \gamma^{\underline{0}}+2 i k_{+} k_{-} \gamma^{\underline{1}}\right] \zeta_{0}=\zeta_{0} .
$$

Given that $\zeta_{0}$ obeys these restrictions, we find that

$$
\begin{aligned}
\bar{\kappa} \kappa & =8 i k^{2} \beta \\
\bar{\kappa} \gamma_{\underline{0}} \kappa & =8 i k^{2} f \\
\bar{\kappa} \gamma_{\underline{1}} \kappa & =0 \\
\bar{\kappa} \gamma_{\underline{2}} \kappa & =8 i k^{2} \sqrt{f^{2}-\beta^{2}},
\end{aligned}
$$

where $k^{2}=\left(k_{+}^{2}+k_{-}^{2}\right)$; these results will prove useful later.

The metric admitting the Killing spinors (2.44) is

$$
d s^{2}=\left((2 m r)^{2}-4 m u+\beta^{2}\right) d t^{2}-2 u d t d \varphi-\frac{\left[1-\left(\frac{u^{\prime}}{4 m r}\right)\right]^{2}}{\left[\left(2 m r-\left(\frac{u}{r}\right)\right)^{2}+\beta^{2}\right]} d r^{2}-r^{2} d \varphi^{2}
$$

Notice that the requirement that there exist Killing spinors leaves undetermined the function $u$ and the constant $\beta$. These are to be determined by the field equations and the boundary conditions.

\section{Supersymmetric vacuum spacetimes}

In section 6 we shall consider the field equations with a sigma model soliton source. Here we shall consider the simpler source-free case. Inserting the metric (2.48) into the source-free Einstein equation (with cosmological constant) we find that

$$
\left(\frac{u^{\prime}}{r}\right)=\text { const. }
$$

By a redefinition $\varphi \rightarrow \varphi+$ const. $\times t$, we can arrange for the integration constant 
on the right hand side of (3.1) to vanish, in which case

$$
u=\frac{1}{2} J
$$

for constant $J$. The metric, with $\alpha=2 m$, is then

$$
d s^{2}=\left[(2 m r)^{2}-2 m J+\beta^{2}\right) d t^{2}-J d t d \varphi-\frac{4 r^{2} d r^{2}}{\left(4 m r^{2}-J\right)^{2}+4 \beta^{2} r^{2}}-r^{2} d \varphi^{2}
$$

By comparison with the black hole solution of [2] we see that $J$ is the total angular momentum of this spacetime, and that the total mass, relative to the black hole vacuum, is $M=2 m J-\beta^{2}$. We shall confirm these identifications in the following section. The gauge-field one-form is

$$
A=-\frac{1}{4 m}(\beta+n) d \varphi
$$

and from (2.40) we see that the associated charge is

$$
Q=-\frac{1}{2}(\beta+n)
$$

Using this relation between $\beta$ and $Q$, the total mass may be expressed in terms of $J$ and $Q$ as

$$
M=2 m J-(2 Q+n)^{2}
$$

Although $\beta$ need not be an integer, one must choose $\beta=-n$ to get $A=0$ and so recover the spacetimes considered previously in the context of the purely Einstein theory. In this case the charge $Q$ vanishes and the metric is

$$
d s^{2}=\left[(2 m r)^{2}-2 m J+n^{2}\right] d t^{2}-J d t d \varphi-\frac{4 r^{2} d r^{2}}{\left(4 m r^{2}-J\right)^{2}+4 n^{2} r^{2}}-r^{2} d \varphi^{2}
$$

When $n=0$ this metric represents a spacetime of mass $M=2 m J$. For positive $J$ (and $n=0$ ) the mass $M$ is positive and the metric is that of the extreme black hole, 
shown previously to admit Killing spinors [3]. A version of this supersymmetric extreme black hole metric with positive mass exists for either sign of $J$ because the transformation $t \rightarrow-t$ in (3.7) effectively changes the sign of $J$ without changing the mass $M$, so the extreme black hole metric may be more generally written as $[2]$

$$
d s^{2}=\left[(2 m r)^{2}-2 m|J|\right] d t^{2}-J d t d \varphi-\frac{4 r^{2} d r^{2}}{\left(4 m r^{2}-|J|\right)^{2}}-r^{2} d \varphi^{2} .
$$

Note that the metric (3.7) continues to admit Killing spinors when $J$ is negative, so the metric

$$
d s^{2}=\left[(2 m r)^{2}+2 m|J|\right] d t^{2}-J d t d \varphi-\frac{4 r^{2} d r^{2}}{\left(4 m r^{2}+|J|\right)^{2}}-r^{2} d \varphi^{2},
$$

with negative mass $M=-2 m|J|$ is also supersymmetric! Unlike the extreme black hole, however, it has a naked cusp-like singularity at $r=0$. For either sign of the mass, the Killing spinor $\kappa$ is given by

$$
\kappa=\sqrt{2 m r-\frac{M}{4 m r}}\left(1-i \gamma^{\underline{1}}\right) \psi_{0}
$$

where $\psi_{0}=\left[\left(k_{-}-k_{+}\right)+\left(k_{-}+k_{+}\right) \gamma^{2}\right] \zeta_{0}$ is a constant spinor. In these cases, the freedom represented by $k_{ \pm}$is clearly not independent of the freedom to choose $\psi_{0}$ and the Killing spinors span a space of real dimension 2. When $M>0$ the Killing spinor $\kappa$ vanishes at $r=\sqrt{M / 8 m^{2}}$, which is the horizon of the black hole. For either sign of $M$ the Killing spinor is singular at $r=0$, but so also is the metric.

Consider now the static spacetimes with $J=0$. The metric is

$$
d s^{2}=\left[(2 m r)^{2}+n^{2}\right] d t^{2}-\frac{1}{\left[(2 m r)^{2}+n^{2}\right]} d r^{2}-r^{2} d \varphi^{2} .
$$

For $n=0$ this is the metric of the black hole vacuum:

$$
d s^{2}=r^{2}\left[d(2 m t)^{2}-d \varphi^{2}\right]-\frac{d r^{2}}{r^{2}}
$$

which would be one form of the adS metric, which has a non-singular Killing horizon at $r=0$, were it not for the fact that $\varphi$ is an angular variable. The identification 
of $\varphi$ with $\varphi+2 \pi$ creates a conical singularity at the horizon, so the black hole vacuum has a null singularity at $r=0$.

Observe that a necessary condition for a Killing spinor $\kappa$ to be non-singular at $r=0$ is that

$$
\kappa \sim r^{\frac{\sigma}{2}} e^{ \pm \frac{i n}{2} \varphi} \kappa_{0} \quad(t=0, r \rightarrow 0)
$$

for some constant (non-zero) spinor $\kappa_{0}$ and non-negative integer $\sigma$. Moreover, if $\sigma=0$ the spinor $\kappa$ will still be singular at $r=0$ unless $|n|=1$.

When $|n|=1$, the metric (3.11) is that of adS space. The corresponding Killing spinors have $\sigma=0$ and are therefore non-singular at $r=0$, as required since the singularity of the metric at $r=0$ is merely a coordinate singularity in this case. When $|n|>1$, the Killing spinors $\kappa$ still have the property (3.13) with $\sigma=0$. They are therefore singular at $r=0$, but this is not problematic because the metric is also singular there. To see this we observe that when $|n|>1$ we may introduce the new variables

$$
\hat{r}=\frac{r}{n}, \quad \hat{\varphi}=n \varphi
$$

in terms of which the metric (3.11) is again adS, but the angular variable $\hat{\varphi}$ is now identified with period $2|n| \pi$ instead of $2 \pi$, which implies a conical singularity at $r=0$ with negative deficit angle $\delta=-2(|n|-1) \pi$. The 'point' $r=0$ must therefore be excluded from the spacetime. It seems that such spacetimes are unphysical.

When $Q \neq 0$ most of the above discussion still applies if $Q$ is half-integral, but with $n$ replaced by $n+2 Q$. Note, however, that although the metric is adS for $J=0$ and $|n+2 Q|=1$, and hence non-singular, the gauge field $A$ is singular at the origin if $Q \neq 0$, so that one cannot have a completely non-singular 'charged' adS spacetime. In contrast, when $J=0$ and $|n+2 Q|=0$, so that the metric is that of the black hole vacuum, it is still possible to have $Q=-\frac{n}{2}$ for $n \neq 0$ without incurring a singularity of the gauge field. This is due to the fact that the spatial sections of the black hole vacuum are not simply connected. 
There is no necessity for $Q$ to be half-integral, however. Consider, for example, the static supersymmetric spacetimes with $J=0$ and $n=0$, for which $Q=-\frac{1}{2} \beta$. Because of the freedom to adjust $n$ we may assume that

$$
0 \leq \beta \leq 1
$$

The metric is

$$
d s^{2}=\left[(2 m r)^{2}+\beta^{2}\right) d t^{2}-\frac{d r^{2}}{\left[(2 m r)^{2}+\beta^{2}\right]}-r^{2} d \varphi^{2}
$$

and the mass is $M=-\beta^{2}=-4 Q^{2}$. These spacetimes have been considered in [4]; they have a conical singularity at the origin with deficit angle $\delta=2 \pi(1-\beta)=$ $2 \pi(1+\sqrt{M})$. They are nevertheless physical because they may form from an initially non-singular shell of matter that collapses to the origin [11]. They may be regarded as point particle solutions and in this sense are not truly vacuum solutions. Here we have shown that these solutions are supersymmetric, for appropriate $U(1)$ charge, in the context of $(2,0)$ adS supergravity.

\section{An energy bound for $(2+1)$ adS gravity}

The total mass and angular momentum of a $(3+1)$-dimensional asymptotically adS spacetime were defined by Abbott and Deser [5] using a modification of the ADM procedure for asymptotically-flat spacetimes. Although the same analysis can be directly taken over for $(2+1)$-dimensional asymptotically-flat spacetimes, a similar but much simpler analysis is available that exploits the Chern-Simons formulation of adS gravity in $2+1$ dimensions.

Consider first an arbitrary semi-simple Lie algebra. Let $K_{\mu}$ be the Lie algebravalued CS gauge field with action

$$
S=\int d^{3} x \operatorname{tr}\left[\varepsilon^{\mu \nu \rho}\left(K_{\mu} \partial_{\nu} K_{\rho}+\frac{2}{3} K_{\mu} K_{\nu} K_{\rho}\right)+K_{\mu} j^{\mu}(\phi)\right],
$$

where $j^{\mu}(\phi)$ is a Lie algebra valued current for the 'matter' fields, $\phi$. The field 
equation of $K_{\mu}$ is

$$
\varepsilon^{\mu \nu \rho} K_{\mu \nu}=-j^{\mu}(\phi)
$$

where $K_{\mu \nu}=\partial_{\mu} K_{\nu}-\partial_{\nu} K_{\mu}$ is the field-strength tensor. We now split $K_{\mu}$ into a background $\bar{K}_{\mu}$ and a (not necessarily small) perturbation $\Delta K_{\mu}$, i.e.

$$
K_{\mu}=\bar{K}_{\mu}+\Delta K_{\mu}
$$

The background is taken to satisfy the source-free equations, i.e.

$$
\bar{K}_{\mu \nu}=0
$$

We can now rewrite the field equation (4.2) as

$$
\varepsilon^{\mu \nu \rho} \bar{D}_{\nu}(\Delta K)_{\rho}=-j_{\text {tot. }}^{\mu}(\phi, \Delta K)
$$

where $\bar{D}$ is the covariant derivative constructed from the background field. Thus, the left hand side of (4.5) is linear in $\Delta K$; the non-linear terms have been moved to the right hand side and included in the 'total' current $j_{\text {tot. }}$. If we now suppose that the background $\bar{K}$ is such as to admit a Lie-algebra valued scalar $\xi$ that is background covariantly constant, i.e.

$$
\bar{D}_{\mu} \xi=0
$$

then the Lie algebra invariant vector density

$$
J^{\mu}=\frac{1}{2 \pi} \operatorname{tr}\left(j_{\text {tot. }}^{\mu} \xi\right)
$$

is conserved, i.e. $\partial_{\mu} J^{\mu}=0$. The field equations in the form (4.5) can now be used 
to re-write this as the identically conserved current

$$
J^{\mu}=-\frac{1}{2 \pi} \varepsilon^{\mu \nu \rho} \partial_{\nu} \operatorname{tr}\left(\Delta K_{\rho} \xi\right)
$$

The associated charge is the surface term

$$
Q(\xi)=-\frac{1}{2 \pi} \oint \operatorname{tr}(\Delta K \xi)
$$

where $\Delta K$ is the one-form with components $\Delta K_{\mu}$ and the integral is taken over a 'circle at infinity'. We can now define $Q(\xi)$ to be the total charge on a spacelike hypersurface associated with a Lie algebra valued scalar that approaches $\xi$ asymptotically. Clearly, $K$ must also approach $\bar{K}$ asymptotically. Thus, the ADM procedure applied to $(2+1)$-dimensional CS gauge theories leads to an identification of charge as the holonomy of an asymptotic $U(1)$ connection.

To apply this result to the $U(1)$ gauge field $A$ of the $(2,0)$ adS supergravity we should take into account that the normalization of the CS action for this field differs from the canonical normalization by a factor of $2 m$. Thus, the associated charge $Q$ is

$$
Q=\frac{m}{\pi} \oint A
$$

as claimed in the previous section. To obtain similar formulae for the energy and angular momentum of asymptotically $(a d S)_{3}$ spacetimes, we recall that the equations of adS gravity are equivalent to CS equations for the $\operatorname{Sl}(2 ; \mathbb{R}) \times S l(2 ; \mathbb{R})$ one-forms,

$$
B_{( \pm)}^{a}=\frac{1}{4} \varepsilon^{a b c} \omega_{a b} \pm m e^{a}
$$

associated with the generators $J^{a} \pm(2 m)^{-1} P^{a}$, where $J^{a}=\frac{1}{2} \varepsilon^{a b c} J_{b c}$ [1]. The normalization of the charges in (4.9) assumes a canonical normalization for the CS action. The field equations of adS gravity are obtainable as the Euler-Lagrange equations of any linear combination of the CS terms for the two $S l(2 ; \mathbb{R})$ factors 
of the adS group, but the standard Einstein-Hilbert form of the action is found by taking $(2 m)^{-1}$ times the difference of the CS terms for $B_{(+)}^{a}$ and $B_{(-)}^{a}[1,6]$. This means that we should identify the charges constructed from $B_{( \pm)}^{a}$ and an appropriately normalized background-covariantly constant future-directed timelike vector $\xi^{a}$ with $\pm 2 m\left[J \pm(2 m)^{-1} E\right]=E \pm 2 m J$, where $E$ is the energy and $J$ is the angular momentum. Thus,

$$
E \pm 2 m J=-\frac{1}{2 \pi} \oint \Delta B_{( \pm)}^{a} \xi_{a}
$$

where

$$
\Delta B_{( \pm)}^{a}=\left(B_{( \pm)}^{a}-\bar{B}_{( \pm)}^{a}\right)
$$

and the adS background gauge potentials $\bar{B}_{( \pm)}^{a}$ are given by

$$
\begin{aligned}
\bar{B}_{( \pm)}^{\frac{0}{ \pm}} & =-m \sqrt{(2 m r)^{2}+1}\left(d t \pm \frac{1}{2 m} d \varphi\right) \\
& =-2 m^{2} r\left[1+\frac{1}{8 m^{2} r^{2}}+O\left(\frac{1}{r^{4}}\right)\right]\left(d t \pm \frac{1}{2 m} d \varphi\right) \\
\bar{B}_{( \pm)}^{\frac{1}{( \pm)}} & =-\frac{m}{\sqrt{(2 m r)^{2}+1}} d r=-\frac{1}{2 r}\left[1+O\left(\frac{1}{r^{2}}\right)\right] d r \\
\bar{B}_{( \pm)}^{2} & =-2 m^{2} r\left(d t \pm \frac{1}{2 m} d \varphi\right) .
\end{aligned}
$$

The background covariantly-constant vector $\xi$ may be expressed in terms of an adS Killing spinor $\kappa$ through the relation

$$
\xi^{a}=-\frac{i}{2} \bar{\kappa} \gamma^{a} \kappa
$$

Note the definition

$$
\bar{\kappa} \equiv i \kappa^{\dagger} \gamma^{\underline{0}}
$$

and the identity

$$
\left(\bar{\kappa} \gamma^{a} \kappa\right)\left(\bar{\kappa} \gamma_{a} \kappa\right) \equiv(\bar{\kappa} \kappa)^{2}
$$

which ensure that $\xi$ is future-directed timelike provided $\bar{\kappa} \kappa$ is non-zero, a condition that is satisfied for the adS background. Using the results of $(2.47)$ with $\beta=1$, we 
find that

$$
\begin{aligned}
& \xi_{0}=4 k^{2} \sqrt{(2 m r)^{2}+1}=8 m r k^{2}+O\left(\frac{1}{r}\right) \\
& \xi_{1}=0 \\
& \xi_{2}=8 m r k^{2} .
\end{aligned}
$$

To fix the constant $k$ we shall evaluate the right hand side of (4.12) for the general black hole solution of [2]. In our notation the black hole metric is

$$
\left[(2 m r)^{2}-M\right] d t^{2}-J d \varphi d t-\frac{d r^{2}}{\left[(2 m r)^{2}-M+\frac{1}{4 r^{2}} J^{2}\right]}-r^{2} d \varphi^{2}
$$

and, as pointed out in [2], the special case of $J=0$ and $M=-1$ is anti-de Sitter spacetime, so we expect that $E=M+1$. Except in the extreme, $M=2 m J$, case, this metric is not included in our ansatz of (2.14) because the factor multiplying $d r^{2}$ in (4.18) is not positive definite for all $r$. However, this factor is positive for large $r$ so we may use our previous results to determine the asymptotic behaviour of $\Delta B_{\varphi}^{a}$. We find that

$$
\begin{aligned}
& \Delta B_{\varphi}^{\frac{0}{\varphi}}=-\frac{M+1}{8 m r}+O\left(\frac{1}{r^{2}}\right) \\
& \Delta B \frac{2}{\varphi}=\frac{J}{4 r}+O\left(\frac{1}{r^{2}}\right) .
\end{aligned}
$$

Combining this with (4.17) we verify the formula (4.12) with $k=1$ and, as expected, $E=M+1$. Let us note here for future reference that, for $t=0$, the asymptotic form of the Killing spinor (2.44) as $r \rightarrow \infty$ is

$$
\kappa \sim \sqrt{2 m r} e^{i \frac{n}{2} \varphi} \kappa_{\infty}
$$

where

$$
\kappa_{\infty}=\left(1-i \gamma^{\underline{1}}\right)\left[\left(k_{-}-k_{+}\right)+\left(k_{-}+k_{+}\right) \gamma^{\underline{2}}\right] \zeta_{0} .
$$

We shall now use the above results to establish a lower bound on the adS energy $E$ following the Witten-Nester proof [15] of the positivity of the ADM energy of asymptotically flat $3+1$ dimensional spacetimes and its generalization to 
asymptotically adS 3+1 dimensional spacetimes [16]. We shall consider asymptotically adS spacetimes admitting a spinor $\chi$ that is asymptotic to one of the Killing spinors found previously. It follows that, asymptotically as $r \rightarrow \infty$,

$$
\begin{aligned}
-\frac{i}{2} \bar{\chi} \gamma^{a} \chi & \sim \xi^{a} \\
-\frac{i}{8} \bar{\chi} \chi & \sim 1 .
\end{aligned}
$$

The precise formulation of the asymptotic condition will be left until later. To avoid the proliferation of \pm signs in the formulae to follow we shall assume that $J$ is non-negative. In this case it will be sufficient to consider the 'modified' Nestertype tensor

$$
\hat{E}^{\mu \nu}=-\frac{i}{4} \bar{\chi} \gamma^{\mu \nu \rho} \mathcal{D}_{\rho} \chi+\text { c.c. }
$$

where $\mathcal{D}$ is the covariant derivative of (2.3). Using (4.14) and (4.22), it is easily seen that (4.12) can be rewritten as

$$
E-2 m J=\frac{1}{4 \pi} \oint d S_{\mu \nu} \hat{E}^{\mu \nu}-\left[\frac{1}{8 \pi} \oint \bar{\chi} \bar{D} \chi+\text { c.c. }\right]-4 Q,
$$

where $\bar{D}$ is the adS background covariant derivative, and $d S_{\mu \nu}$ is the dual of the line element of the circle at infinity.

Assuming now that the circle at infinity is the only boundary of a spacelike two-surface with dual surface element $d S_{\mu}$, we have from Gauss' law that

$$
\begin{aligned}
\frac{1}{4 \pi} \oint d S_{\mu \nu} \hat{E}^{\mu \nu} & =\frac{1}{2 \pi} \int d S_{\mu} D_{\nu} \hat{E}^{\mu \nu} \\
& =-\frac{i}{4 \pi} \int d S_{\mu}\left\{\overline{\mathcal{D}_{\nu} \chi} \gamma^{\mu \nu \rho} \mathcal{D}_{\rho} \chi-e^{-1} \bar{\chi}\left(m G^{\mu}+S^{\mu}{ }_{a} \gamma^{a}\right) \chi\right\}
\end{aligned}
$$

At this point we need the field equations for the metric and the abelian gauge field. In order to allow for sources we take these equations to be

$$
S^{\mu}{ }_{a}=-\frac{1}{2} T^{\mu \nu} e_{a \nu} \quad G^{\mu}=\frac{1}{2 m} J^{\mu},
$$

where $T^{\mu \nu}$ and $J^{\mu}$ are the matter stress tensor and $U(1)$ current respectively. Then 
(4.25) becomes

$$
\frac{1}{4 \pi} \oint d S_{\mu \nu} \hat{E}^{\mu \nu}=-\frac{i}{4 \pi} \int d S_{\mu}\left\{\overline{\mathcal{D}_{\nu} \chi} \gamma^{\mu \nu \rho} \mathcal{D}_{\rho} \chi+\frac{1}{2} e^{-1} \bar{\chi}\left(T^{\mu a} \gamma_{a}-J^{\mu}\right) \chi\right\}
$$

The second term in the integral on the RHS of this equation is non-negative provided that the vector field with components $-i \bar{\chi}\left(T_{a}^{\mu} \gamma^{a}-J^{\mu}\right) \chi$ is non-spacelike and future directed for all spinors $\chi$. This condition is trivially satisfied in the absence of matter and it is also satisfied by the currents of the supersymmetric sigma-model, as we shall show later. The first term on the RHS of (4.27) can be shown to be positive in the standard way provided that the spinor $\chi$ satisfies the Witten-type condition

$$
{ }^{(2)} \mathcal{D} \chi \equiv{ }^{(2)} g^{i j} \gamma_{i} \mathcal{D}_{j} \chi=0
$$

where ${ }^{(2)} g^{i j}$ is the inverse of the spatial 2 -metric.

It can be shown that, at least locally, there exists a solution of (4.28) with the asymptotic form

$$
\chi \sim \sqrt{2 m r} e^{\mp i \nu} \kappa_{\infty} \mp \gamma^{2}\left[\frac{\nu}{2}+Q\right] \frac{e^{\frac{\mp i \nu}{2} \varphi}}{\sqrt{2 m r}} \kappa_{\infty}
$$

for any integer $\nu$. Note that this spinor has the required asymptotic property (4.22). To prove the existence of this solution one writes $\chi=\chi_{\infty}+\chi_{1}$ where $\chi_{\infty}$ equals the right hand side of (4.29) and $\chi_{1}$ is assumed to fall off faster than $1 / \sqrt{r}$ as $r \rightarrow \infty$. One can then prove by a variant of the original argument of [15] that the operator ${ }^{(2)} \not \mathcal{D}$ has no zero eigenvalues on the space of functions with the asymptotic fall off of $\chi_{1}$. It then follows that there exists, at least locally, a solution of the form $\chi=\chi_{\infty}+\chi_{1}$ that is determined by $\chi_{\infty}$. The form of $\chi_{\infty}$ can be determined by solving the Witten condition for large $r$ and the result is that given in (4.29). There may be global obstructions that nevertheless prevent the existence of the required solution of the Witten condition. We shall return to this question below. Given the absence of global obstructions, we have now shown that 
the first term in the integral on the RHS of (4.27) is also non-negative and hence that the LHS is non-negative. Using this information in (4.24), we conclude that

$$
E-2 m J \geqslant\left[\frac{1}{8 \pi} \oint \bar{\chi} \bar{D} \chi+c . c .\right]-4 Q
$$

which can be saturated only if $\mathcal{D} \chi=0$, i.e. only if $\chi$ is a Killing spinor. The line integral of $\bar{\chi} \bar{D} \chi$ may be computed using (4.29). It vanishes only when $\nu=1$ and $Q=0$ and the final result is such that (4.30) is equivalent to

$$
E-2 m J \geqslant 1-(2 Q+\nu)^{2}
$$

By an appropriate change of sign in the covariant derivative in $\hat{E}^{\mu \nu}$ one can also prove this inequality for the opposite sign of $J$; we thus derive the bound

$$
M \geqslant|2 m J|-(2 Q+\nu)^{2}
$$

This is our main result concerning energy bounds in three-dimensional adS spacetime. Note that for $\nu=n$ the bound is saturated by the spacetimes (3.3) labelled by the integer $n$ in section 2. As we saw there, these solutions indeed admit Killing spinors.

Consider the special case for which $Q=0$. In this case the strongest bound on the energy is found by choosing $\nu=0$, in which case we have that

$$
M \geqslant 2 m|J|,
$$

which was also found in [7] by other methods. For $J=0$ this is saturated by the black hole vacuum which admits a Killing spinor having the asymptotic form of (4.29) with $\nu=0$. However, there may be global obstructions to the existence of a solution to the Witten condition that invalidate the bound. In fact, this is necessarily the case since the bound (4.33), for $J=0$, is violated by adS space, 
which is non-singular and has $M=-1$ [2]. Clearly, the assumption of the absence of a global obstruction to the existence of a solution to the Witten condition with the asymptotic behaviour of (4.29) must be false for adS space if one assumes that $\nu=0$. This accords with the fact that the Killing spinor of adS space has the asymptotic form (4.29) but with $\nu=1$. For spacetimes for which $\nu=1$ gives the correct asymptotic behaviour of globally-defined spinors one instead finds the bound $M \geqslant-1$, which is saturated by the adS spacetime.

An instructive (although non-supersymmetric) example is provided by the interior metric of a homogeneous circularly symmetric collapsing dust ball:

$$
d s^{2}=d t^{2}-a(t)^{2}\left[\frac{d r^{2}}{\left(1-r^{2}\right)}+r^{2} d \theta^{2}\right]
$$

The frame one-forms can be chosen to be

$$
e^{\underline{0}}=d t \quad e^{\underline{1}}=\frac{a d r}{\sqrt{1-r^{2}}} \quad e^{\underline{2}}=\operatorname{ard} \varphi
$$

from which we compute that

$$
\begin{aligned}
B_{\underline{0}} & =-m d t+\frac{1}{2} \sqrt{1-r^{2}} d \varphi \\
B_{1} & =a m \sqrt{1-r^{2}} d r \\
B_{\underline{2}} & =a m r d \varphi .
\end{aligned}
$$

The metric is non-singular at $r=0$ and Cauchy surfaces include this point. It follows that the spinor $\chi$ satisfying the Witten condition must be non-singular at $r=0$ and, as stated previously, this restricts $\chi$ to have the behaviour

$$
\chi \sim r^{\frac{\sigma}{2}} e^{i \frac{\nu}{2} \varphi} \chi_{0} \quad(t=0, r \rightarrow 0)
$$

for integer $\nu$, non-negative integer $\sigma$ and non-zero constant spinor $\chi_{0}$. Demanding 
that $\chi$ of this form satisfy (4.28) one finds that

$$
(\sigma+1) \chi_{0}=-\nu \gamma^{\underline{0}} \chi_{0}
$$

which implies that

$$
(\sigma+1)^{2}=\nu^{2}
$$

We see immediately that $\nu=0$ is not possible since $\sigma$ cannot be negative. The lowest admissable value of $|\nu|$ is $|\nu|=1$, which corresponds to $\sigma=0$. Linearity of (4.28) and continuity imply that the phase of $\chi$ as $r \rightarrow \infty$ remains equal $\nu \phi$, so the integer $\nu$ in (4.37) is the same as the integer $\nu$ in (4.29) which, as we have now shown, cannot vanish. This is a satisfactory conclusion because had a non-singular spinor $\chi$ been allowed for $\nu=0$ we could have used it to derive the classical bound $M \geqslant 0$, which we know to be false. As things stand, we may choose $|\nu|=1$ and this allows the derivation of the classical bound $M \geqslant-1$. We consider this example as evidence for the conjecture that global obstructions to solutions of the Witten condition (4.28) never exclude (under the conditions discussed earlier) both $\nu=0$ and $|\nu|=1$. If this is true then the validity of the bound $M \geqslant-1$ extends to the quantum theory. This would establish the absolute stability of the adS vacuum. The status of the black hole vacuum is quite different since it saturates the weaker $\nu=0$ bound $M \geqslant 0$. It seems likely that the black hole vacuum will be unstable to decay by quantum tunelling for rather similar reasons to those that lead to an instability of the $4+1$ dimensional KK vacuum [10]. 


\section{5. $(2,0)$ adS Supergravity sigma models}

The fields of the $N=2$ sigma-model action consist of the scalar fields $\phi^{I}$, which are maps from spacetime to the $2 n$-dimensional target space $\mathcal{M}$, and the complex sigma-model fermions $\lambda^{i},(i=1, \ldots, n) . \mathrm{N}=2$ supersymmetry requires the target space to be Kähler. That is, there must exist a closed two form $\Omega$ whose components are related to a complex structure $\Omega^{I}{ }_{J}$ on $\mathcal{M}$ by

$$
\Omega_{I J} \equiv g_{I K} \Omega^{K}{ }_{J} \quad I, J, K=1,2, \ldots, \operatorname{dim} \mathcal{M}
$$

We note here that $d \Omega=0$ implies that $\Omega_{I J}$ can be expressed locally in terms of a vector $X$ as

$$
\Omega_{I J}=X_{J, I}-X_{I, J} .
$$

The vector $X$ cannot be globally-defined on a compact Kähler manifold because in that case $\Omega$ is not exact. Since the holonomy of the (torsion free) affine connection of a $2 n$-dimensional Kähler manifold is $U(n)$ it is possible to introduce a set of complex frame one-forms $\left\{f^{i}, i=1 \ldots, n\right\}$ in the fundamental representation of $U(n)$, with components $f_{I}{ }^{i}$. Their complex conjugates $\left\{f_{i}\right\}$, with components $f_{I i}$ transform in the $\bar{n}$ representation of $U(n)$. The metric $g$ and two-form $\Omega$ can now be expressed in terms of the components of the frame one-forms, i.e. the vielbein, as follows:

$$
g_{I J}-i \Omega_{I J}=2 f_{I}^{i} f_{J i} .
$$

The introduction of the vielbein is convenient because it allows the supersymmetry transformations involving the sigma-model fields to be expressed in terms of a single complex spinor parameter. Otherwise, the second-supersymmetry transformation,

unlike the first, is expressed in terms of the complex structure and this obscures the fact that both are really on the same footing. 
The inverse complex vielbein $f^{I}$, with complex conjugate $f^{I i}$, is defined by

$$
\begin{aligned}
& f^{I}{ }_{i} f_{I}{ }^{j}=\delta_{i}^{j} \quad f^{I i} f_{I j}=\delta_{j}^{i} \\
& f^{I}{ }_{i} f_{I j}=0 \quad f^{I i} f_{I}^{j}=0
\end{aligned}
$$

and

$$
f^{I}{ }_{i} f_{J}^{i}+f^{I i} f_{J i}=\delta_{J}^{I}
$$

from which it follows that

$$
f^{I}{ }_{i} g_{I J}=f_{J i}
$$

These relations are invariant under local $U(n)$ transformations. To preserve this invariance in the fermion couplings of the sigma model we must introduce an (antihermitian) $U(n)$ connection one-form $L^{i}{ }_{j}$ which is determined in terms of the frame forms by the requirement that

$$
\begin{aligned}
d f^{i}+L^{i}{ }_{j} f^{j} & =0 \\
d f_{i}+f_{j} L^{j}{ }_{i} & =0 .
\end{aligned}
$$

It follows that

$$
F_{j}^{i} f^{j}=f_{i} F^{i}{ }_{j}=0
$$

where

$$
F_{j}^{i}=d L^{i}{ }_{j}+L_{k}^{i} L^{k}
$$

is the curvature two-form. As a consequence of (5.8), it has the property that the tensor

$$
M_{k i}{ }^{\ell j} \equiv f^{I \ell} f_{k}^{J} F_{I J^{j}}{ }_{i}
$$

is symmetric on its upper and lower indices. 
The action for the $\mathrm{N}=2$ supersymmetric sigma model in $2+1$ dimensional Minkowski spacetime is

$$
\begin{gathered}
S=\int d^{3} x\left\{\frac{1}{2} g_{I J} \partial_{\mu} \phi^{I} \partial^{\mu} \phi^{J}-\bar{\lambda}_{i} \gamma^{\mu}\left(\partial_{\mu} \lambda^{i}+\partial_{\mu} \phi^{I} L_{I}{ }^{i}{ }_{j} \lambda^{j}\right)\right. \\
\left.-\frac{1}{6} M_{i j}{ }^{k \ell}\left(\bar{\lambda}_{k} \gamma^{\mu} \lambda^{i}\right)\left(\bar{\lambda}_{\ell} \gamma_{\mu} \lambda^{j}\right)\right\}
\end{gathered}
$$

where the conjugate spinor $\bar{\lambda}_{i}$ is defined by

$$
\bar{\lambda}_{i} \equiv\left(\lambda^{i}\right)^{\dagger} i \gamma^{\underline{0}}
$$

This action is invariant under the supersymmetry transformations

$$
\begin{aligned}
\delta \phi^{I} & =\frac{i}{2} f_{i}^{I}{ }_{i} \overline{\lambda^{i}}+\text { c.c. } \\
\delta \lambda^{i} & =-\frac{i}{2} f_{I}{ }^{i} \gamma^{\mu} \epsilon \partial_{\mu} \phi^{I}-L_{I}{ }^{i}{ }_{j} \delta \phi^{I} \lambda^{j}
\end{aligned}
$$

We now turn to the construction of the action for the $N=2$ sigma-model coupled to $(2,0)$ adS supergravity. We shall use the gamma-matrix and other conventions of [1]. Note that because of our $(+--)$ metric convention the gammamatrices are all pure imaginary. Also

$$
e \gamma^{\mu \nu \rho}=i \varepsilon^{\mu \nu \rho}
$$

where $e=\operatorname{det} e_{\mu}{ }^{a}$ and $\varepsilon^{\mu \nu \rho}$ is a (constant) tensor density. Note also that the constant Lorentz-invariant tensor $\epsilon^{a b c}$ is defined by

$$
\epsilon^{a b c}=e^{-1} e_{\mu}^{a} e_{\nu}^{b} e_{\rho}^{c} \varepsilon^{\mu \nu \rho}
$$

The fact that the two-form $\Omega$ is closed has the consequence that the vector density

$$
j^{\mu}(\Omega)=\frac{1}{2} \varepsilon^{\mu \nu \rho} \partial_{\nu} \phi^{I} \partial_{\rho} \phi^{J} \Omega_{I J}
$$

is identically conserved $\left(\partial_{\mu} j^{\mu}(\Omega) \equiv 0\right)$. It is therefore a potential source for the Chern-Simons gauge field $A$ and the analogy with supergravity/sigma-model ac- 
tions in $4+1$ dimensions suggests that such a term be included. Indeed, the inclusion of such a coupling leads to the following locally-supersymmetric and gaugeinvariant action:

$$
\begin{aligned}
S & =\int d^{3} x\left\{\left[-\frac{1}{2} e R+\frac{i}{2} \varepsilon^{\mu \nu \rho} \bar{\psi}_{\mu} \mathcal{D}_{\nu} \psi_{\rho}-2 m \varepsilon^{\mu \nu \rho} A_{\mu} \partial_{\nu} A_{\rho}+4 m^{2} e\right]\right. \\
& +\frac{1}{2} e g_{I J} \partial_{\mu} \phi^{I} \partial^{\mu} \phi^{J}-A_{\mu} j^{\mu}(\Omega)-\frac{1}{8 m} X_{K} \partial_{\mu} \phi^{K} j^{\mu}(\Omega) \\
& -e \bar{\lambda}_{i} \gamma^{\mu}\left(\nabla_{\mu} \lambda\right)^{i}-\frac{1}{2} e\left[i f_{I}^{i} \bar{\lambda}_{i} \gamma^{\mu} \not \partial \phi^{I} \psi_{\mu}+c . c .\right]-i m e \bar{\lambda}_{i} \lambda^{i} \\
& +\frac{1}{8} e \bar{\lambda}_{i} \lambda^{i}\left(\bar{\psi}_{\nu} \gamma^{\mu} \gamma^{\nu} \psi_{\mu}\right)-\frac{1}{8} e \bar{\lambda}_{i} \gamma^{\rho} \lambda^{i}\left(\bar{\psi}_{\nu} \gamma^{\mu} \gamma_{\rho} \gamma^{\nu} \psi_{\mu}-\bar{\psi} \cdot \gamma \psi_{\rho}-\bar{\psi}_{\rho} \gamma \cdot \psi\right) \\
& \left.-\frac{1}{6} e M_{i j} k \ell\left(\bar{\lambda}_{k} \gamma^{\mu} \lambda^{i}\right)\left(\bar{\lambda}_{\ell} \gamma_{\mu} \lambda^{j}\right)-\frac{1}{4} e\left(\bar{\lambda}_{i} \lambda^{i}\right)^{2}\right\},
\end{aligned}
$$

where the covariant derivative $\nabla$ is defined by

$$
\left(\nabla_{\mu} \lambda\right)^{i}=D_{\mu} \lambda^{i}+\partial_{\mu} \phi^{I} L_{I}{ }^{i}{ }_{j} \lambda^{j}+2 i m A_{\mu} \lambda^{i}
$$

and the spin-connection used to define the Lorentz-covariant derivative $D$ is now taken to be the one for which

$$
D_{[\mu} e_{\nu]}^{a}=\frac{1}{4} \bar{\psi}_{\mu} \gamma^{a} \psi_{\nu}-\frac{i}{4} e_{\mu b} e_{\nu c} \epsilon^{a b c} \bar{\lambda}_{i} \lambda^{i}
$$

That is, the torsion now includes a contribution from the sigma-model fermions.

This action is invariant under the following supersymmetry transformations:

$$
\begin{aligned}
\delta_{\epsilon} e_{\mu}{ }^{a} & =\frac{1}{4} \bar{\epsilon} \gamma^{a} \psi_{\mu}+\text { c.c. } \\
\delta_{\epsilon} \psi_{\mu} & =\mathcal{D}_{\mu} \epsilon-\frac{1}{4} \bar{\lambda}_{i} \gamma^{\nu} \lambda^{i} \gamma_{\mu \nu} \epsilon \\
\delta_{\epsilon} A_{\mu} & =\frac{1}{4}\left(\bar{\epsilon} \psi_{\mu}+\text { c.c. }\right)-\frac{1}{4 m} \delta_{\epsilon} \phi^{I} \partial_{\mu} \phi^{J} \Omega_{I J} \\
\delta_{\epsilon} \phi^{I} & =\frac{i}{2} f^{I}{ }_{i} \bar{\epsilon} \lambda^{i}+\text { c.c. } \\
\delta_{\epsilon} \lambda^{i} & =-\frac{i}{2} f_{I}{ }^{i} \not \partial \phi^{I} \epsilon-L_{J}{ }_{j}{ }_{j} \delta_{\epsilon} \phi^{J} \lambda^{j}
\end{aligned}
$$


where

$$
\hat{\partial}_{\mu} \phi^{I}=\partial_{\mu} \phi^{I}-\left[\frac{i}{2} f_{i}^{I} \bar{\psi}_{\mu} \lambda^{i}+\text { c.c. }\right]
$$

It is also invariant under the gauge transformations

$$
\begin{aligned}
\delta_{\Lambda} \psi_{\mu}=-i \Lambda \psi_{\mu} & \delta_{\Lambda} A_{\mu}=(2 m)^{-1} \partial_{\mu} \Lambda \\
\delta_{\Lambda} \phi^{I}=0 & \delta_{\Lambda} \lambda^{i}=-i \Lambda \lambda^{i},
\end{aligned}
$$

for arbitrary function $\Lambda(x)$.

\section{Supersymmetric self-gravitating solitons}

For present purposes we need only the bosonic sector of the supergravity coupled sigma model action just constructed. This is

$$
\begin{gathered}
S_{\text {bos. }}=\int d^{3} x\left\{-\frac{1}{2} e R-2 m \varepsilon^{\mu \nu \rho} A_{\mu} \partial_{\nu} A_{\rho}+4 m^{2} e+\frac{1}{2} e g_{I J} \partial_{\mu} \phi^{I} \partial^{\mu} \phi^{J}\right. \\
\left.-A_{\mu} j^{\mu}(\Omega)-\frac{1}{8 m} X_{K} \partial_{\mu} \phi^{K} j^{\mu}(\Omega)\right\}
\end{gathered}
$$

We shall also need the fermion supersymmetry transformations in a purely bosonic background. These are

$$
\begin{aligned}
\delta_{\epsilon} \psi_{\mu} & =\mathcal{D}_{\mu} \epsilon \equiv\left(\partial_{\mu}+i B_{\mu}{ }^{a} \gamma_{a}+2 i m A_{\mu}\right) \epsilon \\
\delta_{\epsilon} \lambda^{i} & =-\frac{i}{2} f_{I}^{i} \not \partial \phi^{I} \epsilon
\end{aligned}
$$

where, since the torsion vanishes in a purely bosonic background, $B_{\mu}{ }^{a}$ is given by $(2.5)$.

The curious final 'topological' term in the action (6.1) deserves comment. For the special case of $\mathcal{M}=S^{2}$ it is just the well-known Hopf term [17]. Its presence is 
required by supersymmetry if the coupling of $A_{\mu}$ to the topological current $j^{\mu}(\Omega)$ is included but its coefficient is such that the field redefinition

$$
A_{\mu}^{\prime}=A_{\mu}+\frac{1}{4 m} X_{K} \partial_{\mu} \phi^{K}
$$

leads to the new, and much simpler, action

$$
S_{b}=\int d^{3} x\left\{-\frac{1}{2} e R-2 m \varepsilon^{\mu \nu \rho} A_{\mu}^{\prime} \partial_{\nu} A_{\rho}^{\prime}+4 m^{2} e+\frac{1}{2} e g_{I J} \partial_{\mu} \phi^{I} \partial^{\mu} \phi^{J}\right\}
$$

in which both the Hopf-type term and the coupling of $A$ to the topological current density $j(\Omega)$ are absent. However, this redefinition would complicate the supersymmetry transformations. Written in terms of $A$ (rather than $A^{\prime}$ ) the supersymmetry transformation of $\psi_{\mu}$ is exactly as it was in the absence of matter, apart from $\lambda^{2}$ terms which vanish in a purely bosonic background. This has the consequence that the Killing spinor equation found previously in the context of the pure $(2,0)$ adS supergravity theory is unchanged by the coupling to matter. This is a special feature of the particular model that we have constructed; more general matter couplings exist but they will not be discussed here.

The field equations of the action (6.1) are

$$
\begin{aligned}
S^{\mu}{ }_{a} & =-\frac{1}{2} T^{\mu}{ }_{a} \\
G^{\mu} & =\frac{1}{2 m} J^{\mu}
\end{aligned}
$$

and

$$
\nabla_{\mu} \partial^{\mu} \phi^{J}=0
$$

where

$$
\begin{aligned}
T_{\mu \nu} & =g_{I J}\left(\partial_{\mu} \phi^{I} \partial_{\nu} \phi^{J}-\frac{1}{2} g_{\mu \nu} \partial_{\rho} \phi^{I} \partial^{\rho} \phi^{J}\right) \\
J^{\mu} & =-j^{\mu}(\Omega),
\end{aligned}
$$

and $\nabla$ is the standard spacetime and target space covariant derivative. The field equations (6.5) are precisely of the form assumed earlier in our derivation of energy 
bounds. From the specific form of the currents one may verify that

$$
\gamma^{\nu} T_{\nu}^{\mu}-J^{\mu}=g^{I J}\left(\bar{P}_{I K} \not \partial \phi^{K}\right) \gamma^{\mu}\left(P_{J L} \not \phi^{L}\right)
$$

where

$$
P_{I J}=\frac{1}{2}\left(g_{I J}+i \Omega_{I J}\right)
$$

and $\bar{P}$ is its complex conjugate. It follows, for any spinor $\chi$, that

$$
-i \bar{\chi}\left(\gamma^{\nu} T^{\mu}{ }_{\nu}-J^{\mu}\right) \chi=-i g^{I J} \bar{\Psi}_{I} \gamma^{\mu} \Psi_{J}
$$

where $\Psi_{I} \equiv P_{I J} \not \partial \phi^{L} \chi$. The right hand side of (6.10) is manifestly non-spacelike and future directed, so the condition on the stress tensor and $U(1)$ current required to establish the classical energy bound in section 3 is indeed satisfied by supersymmetric sigma-model matter. As we saw in that section the energy bound can be saturated only if $\chi=\kappa$ where $\kappa$ is a Killing spinor. It is also clear from (4.27) and (6.10) that a further condition for saturation of the bound in the presence of sigma model matter is that, when $\chi=\kappa, \psi_{I}=0$. Not surprisingly, this is equivalent to the condition that $\delta_{\epsilon} \lambda^{i}=0$ if the anti-commuting complex spinor parameter $\epsilon$ is replaced by the complex commuting Killing spinor $\kappa$. Thus, to find supersymmetric non-vacuum spacetimes we must find sigma model fields $\phi^{I}$ such that

$$
P_{I J} \not \partial \phi^{J} \kappa=0
$$

where $\kappa$ is a Killing spinor. Given that $\kappa$ has the form $\kappa=\left(1+b_{a} \gamma^{a}\right) \zeta$, where $b_{a}$ are the functions introduced in section 2 , we find that (6.11) is equivalent to

$$
\frac{1}{2}\left(g_{I J}+i \Omega_{I J}\right) \partial_{\mu} \phi^{J}\left(e_{c}^{\mu}+i \varepsilon_{c}^{a b} e_{a}{ }^{\mu} b_{b}\right)=0
$$

To solve these equations we consider matter fields of the form $\phi^{I}(\varphi+2 m t, r)$ 
and we try the ansatz

$$
\partial_{\varphi} \phi^{I}=\gamma(r) \Omega^{I}{ }_{J} \partial_{r} \phi^{J}
$$

This leads to a solution of (6.12) provided that

$$
\gamma=\frac{r f}{h \beta},
$$

where $\beta$ is the constant introduced in section 2 , and the function $f$ is given by (2.36) (with $\alpha=2 m$ ). Clearly we must exclude $\beta=0$ here but it follows from (2.42) that $G^{\mu}=0$ when $\beta=0$, and then from (6.5) and (6.7) that $\phi^{I}$ must be constant, so no generality is lost by this exclusion.

We have now reduced the solution of the conditions (6.12) for the matter fields to preserve supersymmetry to the problem of finding functions $\phi^{I}(\varphi+2 m t, r)$ satisfying (6.13). If we introduce the complex spacetime coordinate

$$
z=\exp \left[\int \gamma^{-1} d r+i(\varphi+2 m t)\right]
$$

and choose complex coordinates $\phi^{\alpha}(\alpha=1, \ldots, n)$ on the target space, then the complex fields $\phi^{\alpha}(\varphi+2 m t, r)$ become functions of $z$ and $\bar{z}$ and (6.13) reduces to

$$
\frac{\partial \phi^{\alpha}}{\partial \bar{z}}=0 .
$$

which is solved by holomorphic functions of $z$. This is precisely the condition found for sigma-model solitons in flat space.

The equation of motion (6.6) for $\phi^{I}$ is now found to be identically satisfied so the holomorphic functions $\phi^{\alpha}(z)$, as well as the function $u$, must be found from the equations (6.5) which, given the results of section 2 , are found to reduce to the single equation

$$
\frac{\beta^{2}}{2 r f^{3} h}(f h)^{\prime}=g_{I J} \partial_{r} \phi^{I} \partial_{r} \phi^{J} .
$$

Using complex coordinates on the target space, and the fact that the fields $\phi^{\alpha}$ are 
holomorphic functions of $z$, we can rewrite this equation as

$$
\frac{r}{2 h^{3} f}(f h)^{\prime}=g_{\alpha \bar{\beta}}|z|^{2} \partial_{z} \phi^{\alpha} \partial_{\bar{z}} \bar{\phi}^{\bar{\beta}} .
$$

Since the functions $f$ and $h$ are determined in terms of $u$ by (2.36), this equation can be viewed as a second order ODE for $u$ with a source determined by the holomorphic functions $\phi^{\alpha}$. However, the LHS of (6.18) depends only on $r$, i.e. on $|z|$, while this is true of the RHS only for very special holomorphic functions. For simplicity, we shall now restrict the discussion to the simplest possible Kähler target manifold, the Riemann sphere, parametrized by a single complex coordinate $\phi$, in which case (6.18) becomes

$$
\frac{r}{2 h^{3} f}(f h)^{\prime}=\frac{4}{\left(1+|\phi|^{2}\right)^{2}}\left|z \partial_{z} \phi\right|^{2} .
$$

In order that the right hand side be a function of $|z|$ only we are forced to set

$$
\phi=C z^{k}
$$

for some constant $C$ and integer $k$. The simplest case is $k=0$, i.e. constant matter fields. The first non-trivial case is $k=1$ and we shall examine this case in some detail. Setting $\phi=C z$ in (6.19) we have

$$
\frac{r}{2 h^{3} f}(f h)^{\prime}=\frac{4|C z|^{2}}{\left(1+|C z|^{2}\right)^{2}} .
$$

To proceed we use the fact that

$$
\frac{\partial}{\partial r}=\frac{\beta h|z|}{r f} \frac{\partial}{\partial|z|}
$$

to rewrite (6.21) as an ODE with $|z|$ as the independent variable. This ODE can be immediately once-integrated to give

$$
\frac{4}{1+|C z|^{2}}=c+\frac{\beta}{f h},
$$

where $c$ is the integration constant. We note for future reference that $f h$ can be constant only if $C=0$, which corresponds to vanishing matter, or $C=\infty$, which 
is clearly unphysical. Constant $f h$ implies constant $u$, i.e a vacuum solution (as expected for $C=0$ ) and thus $f h=1$ by our analysis of section 2 . It follows that

$$
\begin{gathered}
C=0 \Rightarrow c=4-\beta \\
C=\infty \Rightarrow c=-\beta .
\end{gathered}
$$

Using (6.23) to eliminate $|C z|$ from (6.21) we obtain the ODE

$$
r f^{2}(f h)^{\prime}=-\frac{1}{2} c(c-4)(f h)^{3}-\beta(c-2)(f h)^{2}-\frac{\beta^{2}}{2}(f h) .
$$

Defining the new dependent and independent variables

$$
r^{2}=y \quad \tilde{u}(y)=2 m r^{2}-u(r)
$$

this equation can be simplified to

$$
\left(\tilde{u}^{2}+\beta^{2} y\right) \tilde{u}^{\prime \prime}=-\frac{c(c-4)}{16 m^{2}}\left(\tilde{u}^{\prime}\right)^{3}-\frac{\beta(c-2)}{4 m}\left(\tilde{u}^{\prime}\right)^{2}-\frac{\beta^{2}}{4} \tilde{u}^{\prime}
$$

We have not been able to find an analytic solution of this second order non-linear differential equation, but assuming that $\tilde{u} \sim 2 m r^{2}$, asymptotically as $r \rightarrow \infty$ the asymptotic solution for $u$ takes the form

$$
u \sim-\frac{K}{4 m} \ln r+\frac{1}{2} \tilde{J}+\mathcal{O}\left(\frac{\ln r}{r^{2}}\right) \quad(r \rightarrow \infty)
$$

where $\tilde{J}$ is a constant and

$$
K=(c+\beta)(c+\beta-4)
$$

is another constant. Because of the logarithmic term, the constant $\tilde{J}$ cannot be identified with the total angular momentum unless $K=0$, hence the change in 
notation. For convenience we recall that the metric under discussion takes the form

$$
d s^{2}=\left((2 m r)^{2}-4 m u+\beta^{2}\right) d t^{2}-2 u d t d \varphi-\frac{\left[1-\left(\frac{u^{\prime}}{4 m r}\right)\right]^{2}}{\left[\left(2 m r-\left(\frac{u}{r}\right)\right)^{2}+\beta^{2}\right]} d r^{2}-r^{2} d \varphi^{2}
$$

From (6.28) we see that its asymptotic form is

$$
\begin{aligned}
d s^{2} \sim & {\left[(2 m r)^{2}+K \ln r-2 m \tilde{J}+\beta^{2}+\mathcal{O}\left(\frac{\ln r}{r^{2}}\right)\right] d t^{2} } \\
& -\left\{\left[(2 m r)^{2}+K \ln r-2 m \tilde{J}+\beta^{2}\right]^{-1}+\mathcal{O}\left(\frac{1}{r^{2}}\right)\right\}-r^{2} d \varphi^{2} .
\end{aligned}
$$

The corresponding expressions for the asymptotic behaviour of the fields $A$ and $\phi$ are given by

$$
\begin{aligned}
& A \sim\left[\frac{\beta K}{32 m^{2} r^{2}}+\mathcal{O}\left(\frac{\ln r}{r^{4}}\right)\right] d t \\
& +\left[-\frac{(\beta+n)}{4 m}-\frac{\beta K}{64 m^{3} r^{2}}+\mathcal{O}\left(\frac{\ln r}{r^{4}}\right)\right] d \varphi \\
& \phi \sim C e^{i(\varphi+2 m t)}\left[1-\frac{\beta}{2 m r}+\mathcal{O}\left(\frac{1}{r^{2}}\right)\right] \text {. }
\end{aligned}
$$

The logarithmic term in (6.28) vanishes when $K=0$, i.e. when either $c=-\beta$ or $c=4-\beta$. In fact, all terms in $u$ other than the constant vanish when $c$ takes one these two values, which therefore lead to vacuum solutions. Since $f h=1$ for a vacuum solution (given $\alpha=2 m$ ), we learn that the implications of (6.24) can be reversed. That is, $c=4-\beta$ implies that $C=0$, which we now exclude since we are interested in soliton solutions, and $c=-\beta$ implies that $C=\infty$, which we exclude as unphysical. We conclude that the logarithmic term is necessarily present for self-gravitating solitons, so $K \neq 0$. When $\tilde{J}=0$ the $K=0$ case can be thought of as the limiting case in which the soliton becomes a point particle at the origin, which is then a conical singularity. It is not difficult to see that the logarithmic term is a necessary feature for a spacetime to be non-singular without horizons and we argue below that the spacetimes of the self-gravitating solitons found here indeed have this property. 
From (6.32) we see that the vector potential $A$ tends to a constant as $r \rightarrow \infty$ and the complex scalar field $\phi$ tends to a phase. From this it is easy to see that both the field strength two-form for $A$ and the energy momentum tensor for $\phi$ vanish at infinity. But since $u$ grows logarithmically the metrics of our soliton solutions are not asymptotically anti de Sitter in the sense of [18] so the results of those authors is not applicable. Nor is it clear that the charges defined as in section 4 make sense. However, although neither $M$ nor $J$ is separately well-defined, the linear combination $\tilde{M}=M-2 m J i s$, because the potentially divergent contribution due to the $\ln r$ term in $u$ cancels, and we may as well define this quantity to be the soliton's mass; it saturates the bound $\tilde{M} \geqslant-\beta^{2}$, as in the vacuum case.

Singularities of the metric (2.48) for $u$ given by (6.26) and (6.27) can arise, in principle, either from some component becoming infinite at finite $r$ or because the inverse fails to exist. Any such singularity may be merely a coordinate singularity, of course; this point must be adressed after location of the singularities. A component of the metric can become infinite for finite $r$ only if $u$ becomes infinite for finite $r$, or when $u$ is of the form $u \sim r$ as $r \rightarrow 0$, in which case $g_{r r}$ would become infinite. As we shall see, neither case can occur. Consider first the case $u \rightarrow \infty$ as $r \rightarrow r_{0}$ or, equivalently, $\tilde{u} \rightarrow \infty$ as $y \rightarrow y_{0}=r_{0}^{2}$. In this case it is convenient to reinterpret (6.27) as an equation for $y$ in terms of $\tilde{u}$, in which case (6.27) can be rewritten as

$$
\left(\tilde{u}^{2}+\beta^{2} y\right) y^{\prime \prime}=\frac{c(c-4)}{16 m^{2}}+\frac{\beta(c-2)}{4 m} y^{\prime}+\frac{\beta^{2}}{4}\left(y^{\prime}\right)^{2}
$$

where now $y=y(\tilde{u})$ and $y^{\prime}=d y / d \tilde{u}$. We have to look for solutions $y$ of this equation that go to a constant as $\tilde{u}$ goes to infinity. Let us write the asymptotic behaviour of $y$ as

$$
y=y_{0}+y_{1}(\tilde{u})
$$

where $y_{1}$ goes to zero as $\tilde{u}$ goes to infinity. Neglecting non-leading terms we have (unless $c=0$ or $c=4$ ) that

$$
(\tilde{u})^{2} y_{1}^{\prime \prime} \approx \frac{c(c-4)}{16 m^{2}}
$$


the solution of which is $y_{1} \propto \ln \tilde{u}$, contrary to assumption. For the cases $c=0$ or $c=4$ we instead find the equation

$$
(\tilde{u})^{2} y_{1}^{\prime \prime} \approx y_{1}^{\prime}
$$

the solution of which is again inconsistent with the asymptotic condition for $y_{1}$. We conclude that $u$ is finite for finite $r$. To exclude the other possibility, that $u \sim r$ as $r \rightarrow 0$, we shall suppose that $\tilde{u} \sim y^{\alpha}$, for some constant $\alpha$, as $y \rightarrow 0$. One then discovers that this is consistent with (6.27) for $\alpha=0$ or $\alpha=1$, but not otherwise.

It remains to determine whether the metric is invertible. Since

$$
\operatorname{det} g=r^{2}(f h)
$$

the metric will be singular, apart from the expected singularity at the origin $r=0$ of the polar coordinates, only if $(f h)$ vanishes. It follows from $(6.21)$ that $(f h) \rightarrow-\infty$ as $r \rightarrow \infty$ if $(f h)$ is anywhere negative. As this contradicts the known behaviour $(f h) \rightarrow 1$ as $r \rightarrow \infty$, we conclude that $(f h)$ cannot be negative. Furthermore, (6.21) implies that $(f h)^{\prime}$ must vanish at any point, other than $r=0$, at which $(f h)$ vanishes. Observe now that linearization of (6.25) about a point at which $(f h)$ vanishes leads to the conclusion that $(f h)^{\prime}$ is everywhere negative in some neighbourhood of this point, but this is not possible unless $(f h)$ changes sign there. Since it cannot change sign our supposition that there was a point at which $(f h)=0$ must be false. Thus $r=0$ is the only point at which the metric is singular. As we have seen, the only possible power behaviour of $\hat{u}$ near the origin is $\hat{u} \sim y^{\alpha}$ for $\alpha=0$ or $\alpha=1$. In the latter case (6.27) also determines the coefficient, such that $\tilde{u}=a y+o(y)$, and $a=-\frac{2 m}{c-4} \beta$ (the value $a=-\frac{2 m}{c} \beta$ is also allowed by the equation but we exclude it since $c=4-\beta$ should correspond to $a=2 m$ ). If we require the singularity of the metric at $r=0$ to be a mere coordinate singularity we further discover that the constant $c$ must be chosen such that $\tilde{u}=2 m \beta y+O\left(y^{2}\right)$ (note that this includes adS for $\beta= \pm 1$ ). When $\tilde{u}$ tends to a non-zero constant 
as $r \rightarrow 0$, non-singularity at $r=0$ restricts the next to leading term so that $u \propto 1 \pm 4 m r+O\left(r^{2}\right)$, athough this possibility is excluded by the requirement that $A$ and $\phi$ also be non-singular at $r=0$, as we shall see. We conclude that (6.27) is compatible with a non-singular metric at the origin.

We now turn to the behaviour of the other fields near the origin. From (2.42) we see that non-singularity of $A$ requires that $d A$ vanish at $r=0$. This restricts the value of $n$ : to $n=0$ when $\tilde{u} \sim$ const. as $r \rightarrow 0$ and to $n=-1$ when $\tilde{u} \sim 2 m \beta y$ as $\rightarrow 0$. However, when $\tilde{u} \sim$ const., $|\phi| \sim$ const. as $r \rightarrow 0$, which (since the constant is non-zero) implies that $\phi$ is singular at $r=0$. On the other hand, when $\tilde{u} \sim 2 m \beta y, \phi \mid \sim r$ as $r \rightarrow 0$, so $\phi$ is non-singular at $r=0$. Thus, non-singular sigma model matter requires $|n|=1$. This is to be expected because when $u \sim r^{2}$ as $r \rightarrow 0$, the Killing spinor $\kappa$ tends to a constant and this is compatible with its non-singularity at $r=0$ only for $|n|=1$.

Thus, the behaviour near $r=0$ of the metric, the CS gauge field, and the sigma-model scalars, is compatible with the existence of a non-singular soliton solution admitting Killing spinors with $|n|=1$. Near $r=0$ the metric is of the form (6.30) with

$$
u(r) \sim 2 m(1-\beta) r^{2}+\mathcal{O}\left(r^{4}\right) \quad(r \rightarrow 0) .
$$

To complete the proof that non-singular self-gravitating solitons exists we would need to show that the solutions of (6.27) that behave like (6.38) near the origin match onto solutions with the asymptotic behaviour (6.28) as $r \rightarrow \infty$. The nonlinearity of the equation for $u$ makes this a difficult problem which we have not solved. 


\section{Conclusions}

The work reported here was initially motivated by some similarities between $2+1$ and $4+1$ dimensions. However, it is ultimately the differences that are most striking. One of these is the fact that there is not just one energy bound but at least two. There are, in fact an infinite number of supersymmetric static spacetimes with $M=-n^{2}$, so that supersymmetry alone does not imply a lower bound on the energy, although only two of these (adS spacetime and the black hole vacuum) have non-singular Cauchy surfaces; the spacetimes for $n>1$ all have a naked conical singularity with negative deficit angle. These facts are related to the fact that, like asymptotically Kaluza-Klein spacetimes, spatial infinity is multiply connected. For this reason one must distinguish between periodic and antiperiodic spinors when deriving the BGH bound. Assuming that this distinction is sufficient for spacetimes that are non-singular on some initial Cauchy surface and that solve the Einstein equations, with matter stress tensor satisfying the dominant energy condition, we concluded that the adS spacetime is absolutely stable, quantum mechanically, as well as classically but that the black hole vacuum might be unstable against quantum tunnelling. This deserves futher investigation.

Yet another surprise is that not only do the $M=2 m|J|$ extreme black hole spacetimes admit Killing spinors, as shown in [3], but so also do the spacetimes with $M=-2 m|J|$, although again these negative mass spacetimes have naked cusp-like singularities and are presumably unphysical. The spacetimes associated with point particles [4] with a mass in the range $-1<M<0$ have a naked conical singularity at the origin with deficit angle $\delta=2 \pi(1+\sqrt{M})$. We have shown that these spacetimes are supersymmetric if the particle has a charge $Q$ such that $M=-4 Q^{2}$ or, equivalently

$$
E>1-4 Q^{2}
$$

where $E$ is the energy with respect to the adS vacuum. We have also shown that this relation can be interpreted as the saturation of a Bogomolnyi-type bound by a supersymmetric solution of the field equations. 
The supersymmetric self-gravitating solitons that we have found can be considered as smeared versions of these supersymmetric point particles, although the asymptotic behaviour is quite different. They share some features with selfgravitating instantonic solitons of five-dimensional supergravity. In the latter case, the curved space equations for the Yang-Mills matter reduced to the flat space self-duality equations as a result of particular interactions of the Maxwell field that were required by supersymmetry [9]. In the $2+1$ case we similarly found that the holomorphicity condition for supersymmetric flat space sigma-model solitons is maintained by the coupling to adS supergravity, but there was a further restrictive condition. In fact, the self-gravitating sigma-model solitons are not strictly asymptotic to anti de Sitter spacetime because of a logarithmic terms in the metric. The criteria for a solution of adS gravity to be a bone fide self-gravitating soliton clearly deserves further study. One wonders, for example, whether the solitons we have found could be pair-produced. The same question could reasonably be asked of the extreme black holes.

Finally, those new supersymmetric spacetimes found here for which $Q=0$ can also be considered as solutions of the effective field equations of three-dimensional string theory [19], so that they will have target space duals associated with exactly the same string theory. It will be of interest to see whether any of the singular supersymmetric asymptotically adS spacetimes have non-singular duals.

Acknowledgements: Discussions with Gary Gibbons, Jim Horne, Paul Howe, George Papadopoulos and Alan Steif are gratefully acknowledged. 


\section{REFERENCES}

1. A. Achúcarro and P.K. Townsend, Phys. Lett. 180B (1986) 89; A. Achúcarro, MSc. Thesis, Univ. of the Basque country, Bilbao, (1986).

2. M. Bañados, C. Teitelboim, and J. Zanelli, Phys. Rev. Lett. 69 (1992) 1849; M. Bañados, M. Henneaux, C. Teitelboim and J. Zanelli, Phys. Rev. D48 (1993) 1506.

3. O. Coussaert and M. Henneaux, Phys. Rev. Lett. 72 (1994) 183.

4. S. Deser and R. Jackiw, Ann. Phys. (N.Y.) 153, (1984) 405.

5. L. Abbott and S. Deser, Nucl. Phys. B195 (1982) 76.

6. E. Witten, Nucl. Phys. B311 (1988/1989) 46.

7. P. Menotti and D. Seminara, Energy theorem for $2+1$ dimensional gravity, preprint MIT-CTP 2324, IFUP-TH-33/94.

8. G.W. Gibbons and C.M. Hull, Phys. Lett. 109B (1982) 190.

9. G.W. Gibbons, D. Kastor, L. London, P.K. Townsend and J. Traschen, Nucl. Phys. B, Nucl. Phys. B416 (1994) 850.

10. E. Witten, Nucl. Phys. B195 (1982) 481.

11. R.B. Mann and S.F. Ross, Phys. Rev. D47 (1993) 3319; Y. Peleg and A. Steif, Phase transition for gravitationally collapsing dust shells in $2+1$ dimensions, preprint WISC-MILW-94-TH-26/UCD-PHY-94-40.

12. B. de Wit, H. Nicolai and A.K. Tollsten, Nucl. Phys. B392 (1993) 3.

13. S.J. Gates, Jr., M.T. Grisaru, M. Roček and W. Siegel, Superspace, or One Thousand and One Lessons in Supersymmetry, (Benjamin/Cummings 1983).

14. A.M. Perelomov, Phys. Rep. 174 (1989) 229.

15. E. Witten, Commun. Math. Phys. 80 (1981) 381; J. Nester, Phys. Lett. A83 (1981) 241. 
16. G.W. Gibbons, C.M. Hull and N. Warner, Nucl. Phys. B218 (1983) 545.

17. F. Wilczek and A. Zee, Phys. Rev. Lett. 51 (1983) 2250.

18. J.D. Brown and M. Henneaux, Commun. Math. Phys. 104 (1986) 207.

19. G.T. Horowitz and D. Welch, Phys. Rev. Lett. 71 (1993) 328. 